\title{
Surface Modifications of Metals Induced by Soft X-Ray Laser Pulse Irradiations
}

\author{
Masahiko ISHINO ${ }^{* 1}$, Anatoly Ya. FAENOV ${ }^{* 1,{ }^{* 2}}$, Momoko TANAKA ${ }^{* 1}$, Noboru HASEGAWA ${ }^{* 1}$, Masaharu NISHIKINO ${ }^{* 1}$, \\ Satoshi TAMOTSU ${ }^{* 3}$, Tatiana A. PIKUZ ${ }^{* 1, *_{2}}$, Toshiyuki OHBA ${ }^{* 1}$, Takeshi KAIHORI ${ }^{* 1}$, and Tetsuya KAWACHI ${ }^{* 1}$ \\ ${ }^{* 1}$ Quantum Beam Directorate, Japan Atomic Energy Agency, 8-1-7, Umemidai, Kizugawa, Kyoto 619-0215, Japan, \\ ${ }^{*}$ Joint Institute for High Temperatures, Russian Academy of Science, Moscow 125315, Russia; \\ ${ }^{*}$ Graduate School of Humanities and Science, Nara Women's University, Kitauoya-Nishimachi, Nara 630-8506, Japan.
}

\begin{abstract}
We irradiated the soft x-ray laser (SXRL) pulses having a wavelength of $13.9 \mathrm{~nm}$, the duration time of 7 ps to aluminum $(\mathrm{Al})$, copper $(\mathrm{Cu})$ and gold $(\mathrm{Au})$ surfaces. After the irradiation processes of SXRL beam with laser flux of $\sim 14 \mathrm{~mJ} / \mathrm{cm}^{2}$ for Al case and of $\sim 21 \mathrm{~mJ} / \mathrm{cm}^{2}$ for $\mathrm{Cu}$ and Au cases, the modified surfaces were observed with the visible microscope, the scanning electron microscope, and the atomic force microscope. The surface modifications caused by the SXRL irradiations were clearly seen on the surfaces, and it was found that the conical structures having around $100 \mathrm{~nm}$ in diameters were formed on the Al surface under a single pulse shot. The conical structures were formed in the features with the average depth of about $40 \mathrm{~nm}$, and this value was in accordance with the attenuation length of the SXRL beam for Al. The modified structure on Al surface induced by SXRL pulse irradiations is different from those of $\mathrm{Cu}$ and $\mathrm{Au}$ surfaces. The modified structure formed on Al surface induced by the SXRL pulse exposure is interesting as the newly structure. Hence, the SXRL beam would be a candidate for a tool of micromachining, which enable to fabricate of three dimensional structures with nano-meter size on $\mathrm{Al}$ surface.
\end{abstract}

DOI:10.2961/jlmn.2012.02.0004

Keywords: soft x-ray laser, single shot exposure, ablation, surface modification, conical structure

\section{Introduction}

Laser pulses having short duration of pico-second or femto-second have abilities to make high temperature, high pressure, excited states of materials, and emit rays, as the results of interactions with matters [1]. Among the short pulse lasers, soft x-ray laser (SXRL) is one of interest laser sources not only for scientific study but also for patterning processing, because of its features of highly spatial coherence, short duration, and short wavelength [2].

At Japan Atomic Energy Agency (JAEA), we generate a SXRL beam having a wavelength of $13.9 \mathrm{~nm}$, the duration of 7 ps [3-6]. Since this SXRL beam has a fully spatial coherence [7-9], many application research experiments, such as the speckle pattern measurement and interference pattern measurement are carried out [10-14].

Recently, we have investigated a characteristic and intensity distribution of the focal patterns of SXRL pulses by means of color center formation in a lithium fluoride (LiF) crystal [15]. In this study, we have clearly seen that the permanent surface alterations, i.e. ablated structures, are produced in the focused patterns on LiF surface. Our experiments reveal that the value of ablation threshold obtained with the SXRL pulse is much smaller than those obtained with other lasers having longer pulse durations and/or longer wavelengths [16]. The low ablation threshold of material for the SXRL beam has a possibility of efficient machining by an ablation process and by a lithography method. However, the intense SXRL pulse will break an expensive mask pattern or a substrate very easily. In addition, physics in the ablation due to the SXRL pulse involves the interaction with intense soft X-ray laser and mat- ter, which is essentially different from that with the optical laser and matter, and we have a chance to observe the change in the optical properties (reflectivity and refractivity) and electronic permittivity for the strongly coupled plasmas or transient phase from solid to plasmas.

The obtained result using our SXRL may become a good benchmark for the experiment using XFEL in near future. Hence, we are interested in a pursuit of ablation process or mechanism for material induced by an irradiation of SXRL pulse.

To confirm the appearance of surface modification induced by the SXRL beam exposure, we irradiated the focused SXRL pulses to metal aluminum (Al) surface [17]. This irradiated surface was observed with a scanning electron microscope (SEM) and an atomic force microscope (AFM) to evaluate the modified surface structures.

In this paper, we obtain the surface modification of different metals including $\mathrm{Al}, \mathrm{Cu}$ and $\mathrm{Au}$ surfaces induced by the irradiation of the SXRL pulses. The possibility of nanometer scale machining at all these metal surfaces produced by the SXRL beam will be also mentioned in the end of this article.

\section{Experiments}

To realize the efficient surface machining by the SXRL beam fabrication, the SXRL irradiation experiments were carried out by the use of the SXRL facility at JAEA.

A schematic diagram of the experimental set up is shown in Fig. 1. The experimental set up was composed of three elements, i.e. the SXRL source, condenser mirror, and 


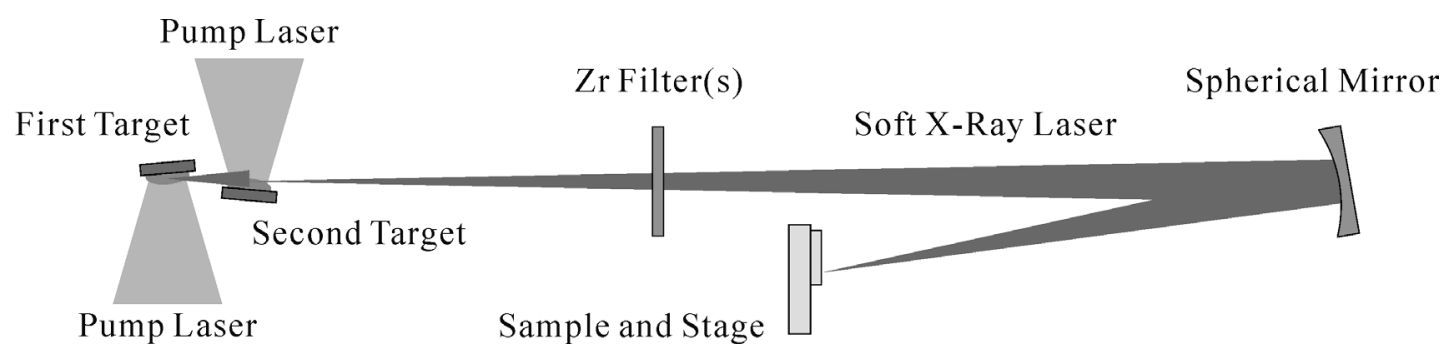

Fig. 1. Schematic diagram of experimental set up for SXRL irradiation.
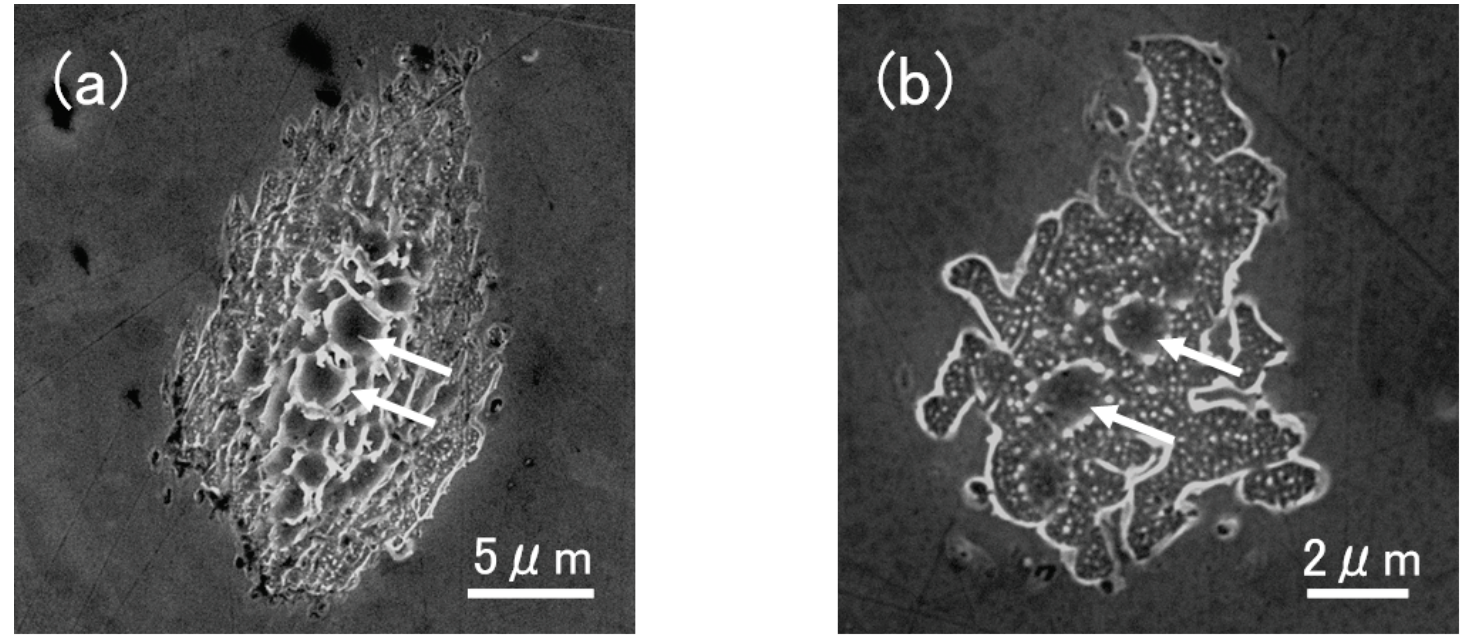

Fig. 2. SEM images of surface modifications on $\mathrm{Al}$ surface induced by the single pulse irradiation of the SXRL beam described with (a) single $\mathrm{Zr}$ filter and (b) two $\mathrm{Zr}$ filters. A $\mathrm{Zr}$ filter with a thickness of $0.2 \mu \mathrm{m}$ has a transmittance of $50 \%$. Then the total fluence at the irradiated surface in Fig. 2(b) was reduced to be half of that in Fig. 2(a).

sample. In this section, we obtain the detail of the SXRL source and of the experimental set up for the SXRL irradiation experiment [17].

\subsection{SXRL source}

The SXRL pulse is generated from the silver $(\mathrm{Ag})$ plasma mediums using an oscillator-amplifier configuration with Ag double targets $[4,5]$. The Ag tapes were used as the plasma targets [18], and the tape targets were irradiated by the linearly focusing laser pulses generated from a chirped pulse amplification glass laser system with zigzag slab Nd:glass amplifiers [19]. The soft x-ray seed light generated by the first Ag plasma was amplified with the second Ag plasma. Characteristics of the generated SXRL from $\mathrm{Ag}$ double targets had a wavelength of $13.9 \mathrm{~nm}$, bandwidth of narrower than $10^{-4}$, duration time of $7 \mathrm{ps}$, and beam divergence of $0.35 \mathrm{mrad}(\mathrm{H}) \times 0.3 \mathrm{mrad}(\mathrm{V})$. The SXRL system worked in $0.1 \mathrm{~Hz}$ regime, and, in addition, the output energy of the order of micro Joules was achieved.

\subsection{SXRL irradiation}

The SXRL pulse was focused on the sample surface by using a Mo/Si multilayer coated spherical mirror having a radius of $1000 \mathrm{~mm}$ in curvature. The output energy of the SXRL pulse was varied in each shot, but the average output energy of the SXRL pulse was estimated to be about $200 \mathrm{~nJ}$.
The Mo/Si multilayer coating was optimized for SXRL at an incidence angle of 2 degrees, and the spherical mirror placed at a distance of $2637 \mathrm{~mm}$ from the SXRL source. A $\mathrm{Zr}$ filter having $0.2 \mu \mathrm{m}$ thick was placed in front of the spherical mirror to reduce the scattered optical radiations from the laser produced Ag plasmas. The transmittance of the $\mathrm{Zr}$ filter and the reflectivity of the $\mathrm{Mo} / \mathrm{Si}$ mirror for the SXRL were about $48 \%$ and about $50 \%$, respectively. Therefore, the total energy of the SXRL beam on the sample surface was about $48 \mathrm{~nJ}$.

Al plate with a thickness of $1 \mathrm{~mm}, \mathrm{Cu}$ plate with a thickness of $0.2 \mathrm{~mm}$, and Au plate with a thickness of 0.5 $\mathrm{mm}$ were used as the targets, and the each target was mounted on the surface of LiF crystal. The targets were moved after each shot along the SXRL beam propagation direction and also perpendicular to it, in order to record the beam patterns on the fresh surface.

LiF crystal was used as an irradiation detector for determination of SXRL intensity distribution in focusing spot $[15,16]$. Target surfaces of $\mathrm{Al}, \mathrm{Cu}$, and $\mathrm{Au}$ were synchronized with $\mathrm{LiF}$ crystal surfaces to assure equal focusing positions (or conditions). The SXRL flux estimated from $\mathrm{LiF}$ detectors were $\sim 14 \mathrm{~mJ} / \mathrm{cm}^{2}$ for Al case and $\sim 21 \mathrm{~mJ} / \mathrm{cm}^{2}$ for $\mathrm{Cu}$ and $\mathrm{Au}$ cases.

All the irradiation process was carried out in a vacuum chamber. 

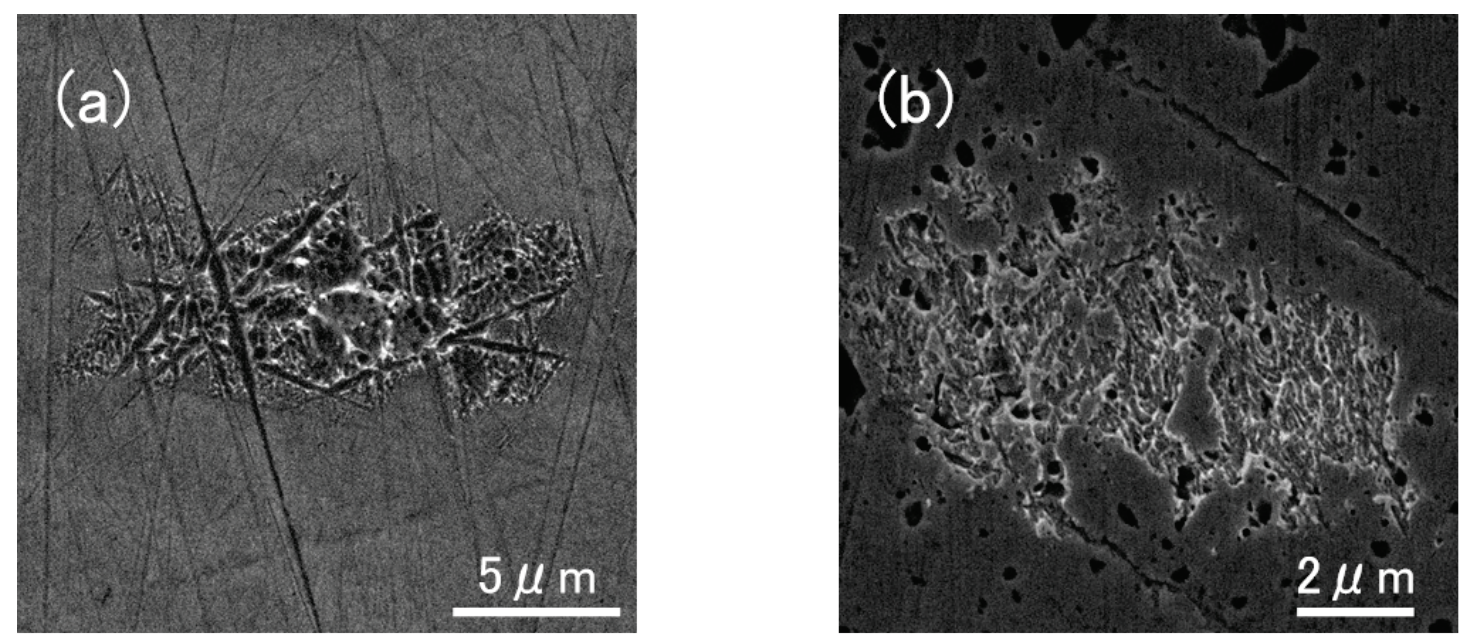

Fig. 3. SEM images of surface modifications (a) on $\mathrm{Cu}$ and (b) on $\mathrm{Au}$ surfaces induced by the single pulse irradiation of the SXRL beams with a single $\mathrm{Zr}$ filter.

\section{Result and discussion}

Figure 2(a) shows the irradiated surface after a single pulse shot of the SXRL beam near the best focal position observed by SEM. The surface modification caused by the single pulse SXRL irradiation, which is different from the original surface, is clearly observed. The area of feature estimated from the SEM image is about $24 \mu \mathrm{m} \times 12 \mu \mathrm{m}$. The distribution of modified feature represents that of the gain structures in Ag plasmas, because the spherical mirror reconstructs a reduction image of the SXRL source. At the center of modified surface, several ablation holes are seen. From the result of AFM measurement, the depths of the ablation holes shown with arrows are measured to be 460 $\mathrm{nm}$ and $410 \mathrm{~nm}$, respectively. These values are not contradictory to our previous work [17]. The single pulse irradiation of the SXRL beam has an ability to create the ablation holes with depths of about $400 \mathrm{~nm}$ on Al surface. At the surrounding areas of the ablation holes, it can be seen that the conical structures are formed. The conical structures are formed in areas of lower fluence region. We have evaluated that the conical structures have a diameter of around 100 $\mathrm{nm}$, and these structures are formed in the shallow features having an average depth of $40 \mathrm{~nm}$, which is in accordance with the attenuation length of SXRL beam to $\mathrm{Al}$ [17].

The irradiated surfaces of copper $(\mathrm{Cu})$ and gold $(\mathrm{Au})$ by the SXRL pulses were also observed. Figures 3(a) and 3(b) show the SEM images of $\mathrm{Cu}$ and $\mathrm{Au}$ surfaces after a single pulse shot of the SXRL beam, respectively [20]. The experimental conditions of the $\mathrm{Cu}$ and $\mathrm{Au}$ surfaces were the same with that of Al surface shown in Fig. 2(a). However, the modified structures induced on $\mathrm{Cu}$ and $\mathrm{Au}$ surfaces were different from that on $\mathrm{Al}$ surface. The conical structures do not exist on $\mathrm{Cu}$ and $\mathrm{Au}$ surfaces, but the ripple-like structures were formed. The differences in surface behaviors would be attributed to the difference of the melting points of materials. The melting points of $\mathrm{Cu}(1357 \mathrm{~K})$ and $\mathrm{Au}(1337 \mathrm{~K})$ are quite higher than that of $\mathrm{Al}(933 \mathrm{~K})$. The theoretical discussions about the modification of Au surface induced by the SXRL beam irradiation have been carried out in the other literatures [21,22].
Anyway, the modified structure formed on $\mathrm{Al}$ surface induced by the SXRL pulse exposure is quite particular structure. The conical structures were formed in the relatively low fluence region, hence it might be possible to increase the area of conical structures by reducing the total energy of the SXRL beam irradiated on Al surface. To reduce the fluence of SXRL pulse irradiation on the Al surface, an additional $\mathrm{Zr}$ filter was inserted in the SXRL axis. Figure 2(b) shows the SEM image of the irradiated surface after a single pulse shot of the SXRL beam with two $\mathrm{Zr}$ filters. The stage position was the same as that in Fig. 2(a). The total energy of the SXRL beam on Al surface was reduced to be $24 \mathrm{~nJ}$. Hence, the depths of the ablation holes is smaller (less than $170 \mathrm{~nm}$ ) than those described with single $\mathrm{Zr}$ filter. The area of modified surface is about $12 \mu \mathrm{m} \times 6$ $\mu \mathrm{m}$. However, the area of surface modification induced by the SXRL pulse become smaller, the relatively area of the conical structures could be increased. In addition, the diameters of formed conical structures, shown in Fig. 2(b), became uniform with each other, so that we would be able to change the size or the dispersion of the conical structures by controlling the fluence of SXRL beam irradiating on $\mathrm{Al}$ surface.

The nano-meter size conical structures induced by the SXRL single pulse irradiation are interesting in the nanometer size surface fabrications. The surface modifications including the conical structures can increase the surface area. One can use as a small catalysts for chemical reactions, when the surface is coated with catalysis materials such as molybdenum, palladium, platinum, and so on. A nanopillar sheet for a culturing of biological cell [23] might to be a candidate of application.

The nano-meter size structures appearing on $\mathrm{Al}$ surface will also be applied to $\mathrm{x}$-ray optics. It is easy to find that the conical structures induced by the SXRL pulse irradiation work as a diffuser or scatter of $\mathrm{x}$-rays. The silicon nitride $\left(\mathrm{Si}_{3} \mathrm{~N}_{4}\right)$ membrane having a few hundred nano-meter thick is able to be use for a transmittable substrate in the large portion of $\mathrm{x}$-ray region. In addition, it is possible to deposit the thin metal films having various thicknesses on 


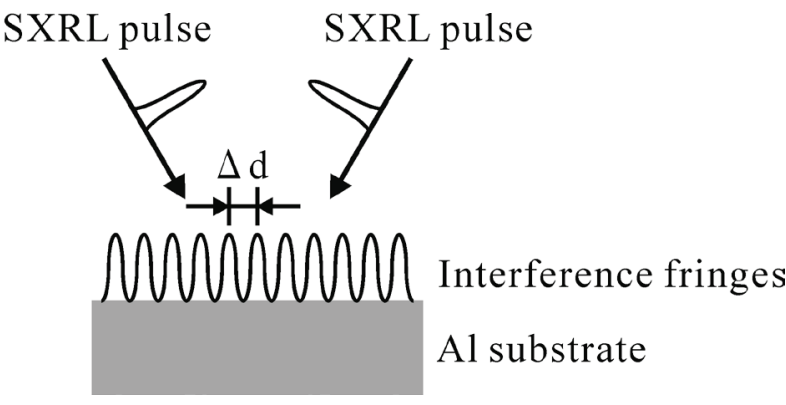

Fig. 4. Schematic design diagram of an interference with the double SXRL pulse interference [26]. $\Delta d$ is the periodic length of interference fringe patterns.

the $\mathrm{Si}_{3} \mathrm{~N}_{4}$ membranes [24]. When one makes the conical structures on the thin Al film, which is deposited on the $\mathrm{Si}_{3} \mathrm{~N}_{4}$ membrane, the structures will work as a kind of transmission-type light shaping diffuser [25] for x-rays.

If we construct an interferometer with double pulses interference (see Fig. 4) [26], a periodical structure including the conical structures will appear on $\mathrm{Al}$ surface. This periodical structure would work as diffraction optics for x-ray region, e.g. direct patterning of the diffraction gratings having extreme short periodic length. The bright parts of interference fringe patterns appear periodically, i.e.

$$
\Delta d=\frac{\lambda}{2 \sin \theta},
$$

where $\lambda$ is the wavelength of SXRL beam $(13.9 \mathrm{~nm})$ and $\theta$ is the incident angle with respect to surface normal of Al. When the incident angles of SXRL beams are set to be less than $4^{\circ}, \Delta d$ will be larger than $100 \mathrm{~nm}$. In this case, the conical structures will appear in the bright parts of fringe patterns, because the diameters of the conical structures are around $100 \mathrm{~nm}$. The original surface will remain on the dark parts of fringe patterns. When the mirror materials such as $\mathrm{Au}$ film or the multilayer mirrors [27] are deposited on the constructed periodical structure, the original (dark) parts of fringes reflect x-rays. Then, this periodical structure works as the x-ray diffraction grating. When the incident angles of SXRL pulses are set to be $\theta=45^{\circ}, \Delta d$ will be about $10 \mathrm{~nm}$. This value corresponds to $10^{5}$ lines $/ \mathrm{mm}$, and it can be very high density grating. In this case, the conical structures may not appear in the irradiated parts of $\mathrm{Al}$ surface, but the modified structures are expected to be formed in the fringe patterns.

It is possible to make the varied-line-space type diffraction grating by use of additional optics to make the spherical and/or aspherical wavefronts [28]. If one wants a transmission grating, it can be fabricate in the same manner of transmission-type light shaping diffuser mentioned above.

When we can control the surface modification process by the SXRL pulse irradiation on Al surface, the SXRL beam would be a candidate for a tool of micromachining, which enable us to fabricate of three dimensional nanometer size structures.

\section{Conclusion}

We irradiated $\mathrm{Al}, \mathrm{Cu}$, and $\mathrm{Au}$ surfaces with the SXRL pulses with a wavelength of $13.9 \mathrm{~nm}$ and a duration time of 7 ps. At the irradiated surfaces, we found that the modified structures including ablation holes were formed by single shot exposure of the SXRL pulse.

In the case of $\mathrm{Al}$ surface, the conical structures having the diameters of around $100 \mathrm{~nm}$ and depth of about $40 \mathrm{~nm}$ were formed in the modified surfaces corresponding with low fluence areas. The ratio of the area forming the conical structures in the modified feature could increase by reducing the total energy of the SXRL pulse.

In the cases of $\mathrm{Cu}$ and $\mathrm{Au}$ surfaces, the ripple-like structures were formed on the irradiated surfaces. The conical structures, which formed on Al surface, did not appear. The differences in surface modifications between Al case and $\mathrm{Cu}$ or $\mathrm{Au}$ cases would be attributed to the difference in the melting points of materials.

The result we demonstrated in this work will be important not only for a pursuit of ablation process but also for future application of SXRL beam such as micromachining.

We discussed the possibilities of fabrications of x-ray diffraction optics by use of the SXRL beam. The wavelength of the SXRL beam is shorter than those of visible lights, and then, potentially the SXRL beam has an ability to draw small patterns, such as grooves or channels with nano-meter order width, on material surface.

\section{Acknowledgments}

This work was partly supported by Grant-in-Aid for Scientific Research (B), No. 21360364 (2009), from the Ministry of Education, Culture, Sports, Science, and Technology (MEXT), Japan. This work was also partly supported by RAS Program No. 22.

The authors are grateful to Dr. M. Yamagiwa for his interest and encouragement on this work.

\section{References}

[1] See for example, J. J. Duderstadt and G. A. Moses, "Inertial Confinement Fusion", (John Wiley \& Sons Ltd., 1982); I. C. E. Turcu, J. B. Dance, "X-rays from Laser Plasmas", (John Wiley \& Sons Ltd., 1998).

[2] P. Jaeglé, "Coherent sources of XUV radiation: Soft Xray lasers and high-order harmonics generation", Springer Series in Optical Science 106 (Springer Science+Business Media, Inc., 2006).

[3] T. Kawachi, M. Kado, M. Tanaka, A. Sasaki, N. Hasegawa, A. V. Kilpio, S. Namba, K. Nagashima, P. Lu, K. Takahashi, H. Tang, R. Tai, M. Kishimoto, M. Koike, H. Daido, and Y. Kato, Phys. Rev. A 66, 033815 (2002).

[4] M. Tanaka, M. Nishikino, T. Kawachi, N. Hasegawa, M. Kado, M. Kishimoto, K. Nagashima, and Y. Kato, Opt. Lett. 28, 1680 (2003).

[5] M. Nishikino, N. Hasegawa, T. Kawachi, H. Yamatani, K. Sukegawa, and K. Nagashima, Appl. Opt. 47, 1129 (2008).

[6] N. Hasegawa, T. Kawachi, T. Utsumi, A. Sasaki, M. Tanaka, M. Kado, K. Sukegawa, P. Lu, M. Kishimoto, R. Tai, K. Nagashima, M. Koike, H. Daido, and Y. Kato, Jpn. J. Appl. Phys. 43, 2519 (2004).

[7] M. Nishikino, M. Tanaka, K. Nagashima, M. Kishimoto, M. Kado, T. Kawachi, K. Sukagawa, Y. Ochi, N. Ha- 
segawa, and Y. Kato, Phys. Rev. A 68, 061802(R) (2003).

[8] M. Nishikino, M. Tanaka, Y. Ochi, M. Kishimoto, M. Ishino, N. Hasegawa, M. Kado, K. Sukagawa, T. Kawachi, and K. Nagashima, IEEE J. Sel. Top. Quantum Electro. 10, 1382 (2004).

[9] Y. Ochi, T. Kawachi, N. Hasegawa, A. Sasaki, K. Nagashima, K. Sukegawa, M. Kishimoto, M. Tanaka, M. Nishikino, and M. Kado, Appl. Phys. B 78, 961 (2004).

[10] R. Z. Tai, K. Namikawa, M. Kishimoto, M. Tanaka, K. Sukegawa, N. Hasegawa, T. Kawachi, M. Kado, P. Lu, K. Nagashima, H. Daido, H. Murayama, A. Sawada, M. Ando, and Y. Kato, Phys. Rev. Lett. 89, 257602 (2002).

[11] R. Z. Tai, K. Namikawa, A. Sawada, M. Kishimoto, M. Tanaka, P. Lu, K. Nagashima, H. Murayama, and M. Ando, Phys. Rev. Lett. 93, 087601 (2004).

[12] H. Tang, O. Guilbaud, G. Jamelot, D. Ros, A. Kilisnick, D. Joyeux, D. Phalippou, M. Kado, M. Nishikino, M. Kishimoto, K. Sukegawa, M. Ishino, K. Nagashima, and H. Daido, Appl. Phys. B 78, 975 (2004).

[13] K. Namikawa, M. Kishimoto, K. Nasu, E. Matsushita, R. Z. Tai, K. Sukegawa, H. Yamatani, H. Hasegawa, M. Nishikino, M. Tanaka, and K. Nagashima, Phys. Rev. Lett. 103, 197401 (2009).

[14] T. Suemoto, K. Terakawa, Y. Ochi, T. Tomita, M. Yamamoto, N. Hasegawa, M. Deki, Y. Minami, and T. Kawachi, Opt. Express 18, 14114 (2010).

[15] A. Ya. Faenov, Y. Kato, M. Tanaka, T. A. Pikuz, M. Kishimoto, M. Ishino, M. Nishikino, Y. Fukuda, S. V. Bulanov, and T. Kawachi, Opt. Lett. 34, 941 (2009).

[16] A. Ya. Faenov, N. A. Inogamov, V. V. Zhakhovskii, V. A. Khokhlov, K. Nishihara, Y. Kato, M. Tanaka, T. A. Pikuz, M. Kishimoto, M. Ishino, M. Nishikino, T. Nakamura, Y. Fukuda, S. V. Bulanov, and T. Kawachi, Appl. Phys. Lett. 94, 231107 (2009).

[17] M. Ishino, A. Ya. Faenov, M. Tanaka, N. Hasegawa, M. Nishikino, S. Tamotsu, T. A. Pikuz, N. A. Inogamov, V. V. Zhakhovsky, I. Yu. Skobelev, V. E. Fortov, V. A. Khohlov, V. V. Shepelev, T. Ohba, T. Kaihori, Y. Ochi, T. Imazono, and T. Kawachi, J. Appl. Phys. 109, 013504 (2011).

[18] M. Nishikino, Y. Ochi, N. Hasegawa, T. Kawachi, H. Yamatani, T. Ohba, T. Kaihori, and K. Nagashima, Rev. Sci. Instrum. 80, 116102 (2009).

[19] Y. Ochi, T. Kawachi, N. Hasegawa, M. Nishikino, T. Ohba, M. Tanaka, M. Nishikino, T. Kaihori, K. Nagashima, and A. Sugiyama, Jpn. J. Appl. Phys. 48, 120212 (2009).

[20] (in preparation for submission.)

[21] S. V. Starikov, V. V. Stegailov, G. E. Norman, V. E. Fortov, M. Ishino, M. Tanaka, N. Hasegawa, M. Nishikino, T. Ohba, T. Kaihori, Y. Ochi, T. Imazono, T. Kawachi, S. Tamotsu, T. A. Pikuz, I. Yu. Skobelev, and A. Ya. Faenov, JETP Lett. 93, 642 (2011).

[22] G. Norman, S. Starikov, V. Stegailov, V. Fortov, I. Skobelev, T. Pikuz, A. Faenov, M. Ishino, M. Tanaka, N. Hasegawa, M. Nishikino, S. Tamotsu, Y. Kato, T. Ohba, T. Kaihori, Y. Ochi, T. Imazono, Y. Fukuda, M. Kando, and T. Kawachi, submitted to Opt. Lett.
[23] S. Nomura, H. Kojima, Y. Ohyabu, K. Kuwabara, A. Miyauchi, and T. Uemura, Jpn. J. Appl. Phys. 44, L1184 (2005).

[24] M. Ishino, M. Kado, M. Nishikino, K. Shinohara, S. Tamotsu, K. Yasuda, N. Hasegawa, M. Kishimoto, T. Ohba, and T. Kawachi, Proc. SPIE 7589, 75891B (2010).

[25] S. Kim, Y. Choi, Y. Ham, C. Park, and J. Kim, Appl. Opt. 42, 2482 (2003).

[26] T. Kawachi, Grant-in-Aid for Scientific Research (B), No. 21360364 (2009) (in Japanese).

[27] See for example, E. Spiller, "Soft X-Ray Optics", (SPIE Optical Engineering Press, 1994); D. Attwood, "Soft X-Rays and Extreme Ultraviolet Radiation, Principles and Applications", (Cambridge University Press, 1999).

[28] T. Namioka, "Vacuum Ultraviolet Spectroscopy I", Experimental Methods in the Physical Science 31, J. A. Samson and D. L. Edere ed., (Academic Press, 1998), Chap. 17.

(Received: June 03, 2011, Accepted: March 08, 2012) 